\title{
Evaluation of androgen receptor status in urothelial carcinoma of the urinary bladder in Egyptian patients: an immunohistochemical study
}

\author{
Mohamed Wagih ${ }^{1^{*}}$ and Marwa Kamel ${ }^{2}$
}

\begin{abstract}
Background: The roles of androgen and androgen receptor (AR) signaling in the oncogenesis of prostate cancer are very well established. What is more poorly understood is the role of AR in other human malignancies including bladder cancer. The incidence of bladder cancer is much higher in males than females, but the exact etiology has not been fully elucidated. This gender disparity has raised the possibility of the AR pathway being involved in the genesis of this disease. Thereby, the aim of this work was to evaluate the expression of AR in a group of Egyptian patients with urothelial bladder carcinoma and to assess whether its expression was correlated with other pathological tumor features. Urothelial bladder carcinoma tissue samples from 50 patients were studied by immunostaining for AR expression in tumor cells.
\end{abstract}

Results: AR was positively expressed in 29 (58\%) patients, while negative expression was observed in 21 (42\%) patients. No statistically significant difference in AR expression with respect to tumor grade $(P=0.07)$ and $p T$ stage $(P=0.09)$ was observed.

Conclusions: The results obtained in this study indicates a clinical value of the AR expression in Egyptian patients with urothelial bladder carcinoma, providing the basis for further studies to evaluate its role and the possibility of new target-based therapies for urothelial bladder carcinoma.

Keywords: Urothelial carcinoma, Androgen receptor, Immunohistochemistry

\section{Background}

Bladder cancer is a common cancer with a prevalence of more than 1,300,000 patients worldwide [1]. In Egypt, bladder cancer represents a massive health burden where it is one of the commonest cancers representing $6.9 \%$ in both sexes and $10.7 \%$ among men. Its distribution is $8.8 \%$ in lower Egypt, 14.2\% in middle Egypt and 12.6\% in upper Egypt. The main risk factor for bladder cancer in Egypt is attributed to urinary schistosomiasis, and despite the

\footnotetext{
*Correspondence: mohamwagih@hotmail.com

1 Department of Pathology, Faculty of Medicine, Beni-Suef University,

Beni-Suef 62521, Egypt

Full list of author information is available at the end of the article
}

continuous efforts for its control, its effect on bladder cancer is still obvious [2].

Urothelial carcinoma is the predominant histological subtype of urinary bladder cancer with $70-80 \%$ being non-muscle invasive disease at the time of diagnosis [3]. Patients initially with non-muscle invasive tumors usually show favorable prognosis. However, those with high-grade cancers have a comparatively higher risk of tumor recurrence and advance to muscle invasion after transurethral resection even with existing intravesical pharmacotherapy. On the other side, patients with muscle invasive tumors often undergo disease progression or metastasis in spite of enduring aggressive procedures, as radical cystectomy with or without systemic 
chemotherapy [4]. Given the frequency and the relapsing nature of urothelial bladder cancer, it is obvious that it represents an enormous load on health-care systems [5]. Therefore, identification of main factors contributing to bladder cancer growth is crucially needed. This might in turn offer unique tumor markers and innovative targeted therapies for bladder cancer patients.

Data indicate that the largest sex discrepancies are for cancers of the esophagus, larynx and bladder, for which the incidence and death rates are about fourfolds higher in men [6]. Even though males display a markedly greater risk for cancer of the bladder, women have a tendency to more aggressive tumors [1]. Environmental factors, such as chemicals and cigarette smoke, were thought to be responsible for this gender-specific difference. However, bladder cancer remains predominant in males even after controlling such factors [7].

Some studies proposed that the hormonal factors play a part in the gender discrepancy in the incidence and behavior of bladder tumors [8]. To support this, some experimental animal studies showed that the development of chemically induced bladder cancer was less in female animals than in males. Additionally, these animal studies generally demonstrate that hormonal changes may modify the tumors and that animals who have been treated with androgen inhibitors had improved survival and more favorable courses [9]. This suggests that androgen may have a role in bladder tumorigenesis. However, the role of androgen in bladder carcinogenesis has been less well studied in humans.

\section{Objectives}

The objectives of the current study therefore were to determine whether or not AR exists in urothelial carcinoma of the urinary bladder of Egyptian patients and to correlate its expression with other pathological parameters.

\section{Methods}

This retrospective study included 50 samples from Egyptian patients with urothelial bladder carcinoma obtained through the collection of archived paraffin blocks of transurethral resection and radical cystectomy specimens from the Pathology department, Faculty of Medicine, as well as a private center during the period from January 2017 till August 2017.

Hematoxylin- and Eosin-stained section was prepared from each block to evaluate the type, grade and stage of the tumor.

The tumors were graded according to the criteria of WHO grading system [10] and staged with respect to the American Joint Committee on Cancer TNM Classification [11].

\section{Immunohistochemistry}

Immunohistochemistry was performed using the Avidin Biotin-Peroxidase complex method. The primary antibody used was the anti-AR monoclonal antibody, clone ENR09 (Dako, Glostrup, Denmark) diluted at a 1:60. The prepared tissue sections were fixed on polyL-lysine coated slides and left to dry overnight. Then, they were dewaxed in xylene and rehydrated graded alcohol series. The sections were immersed in $3 \%$ hydrogen peroxide for $5 \mathrm{~min}$ to inhibit the endogenous peroxidase activity. For antigen retrieval, the sections were heated in a microwave for $15 \mathrm{~min}$ and then they were cooled for $30 \mathrm{~min}$ and washed with phosphate buffer saline (PBS). Next, a blocking serum was added for $20 \mathrm{~min}$ followed by incubating the sections with the primary antibody. Then, the sections were treated with the secondary antibody for $20 \mathrm{~min}$ and incubated with the peroxidase conjugated streptavidin (Dako) for $30 \mathrm{~min}$. Afterward, 3,3-diaminobenzedine tetrahydrochloride (DAB, Dako) was added as a chromogen and finally, the sections were counterstained with hematoxylin, dehydrated and cover slipped.

A tissue section of benign prostatic epithelium was used as a positive control, while the negative control was prepared by omitting the primary antibody and adding PBS instead.

The sections were examined for the presence of brown nuclear staining of AR in tumor cells. The percentage of positive tumor cells was determined in 10 random areas at X400 magnification, and AR expression was classified as: Negative $(<10 \%$ positive tumor cells $)$ and positive ( $\geq 10 \%$ positive tumor cells) [12].

\subsection{Statistical analysis}

Data were collected and analyzed using SPSS statistical package, version 11.5 (SPSS Inc., Chicago, Illinois, USA), and a $P$ value less than 0.05 was considered the cutoff value for significance. Chi-squared test was used to evaluate the relationship between AR expression and the studied parameters.

\section{Results}

The clinical and pathologic features of the studied cases are displayed in Table 1 . The study involved 38 (76\%) male and 12 (24\%) female patients. Ages ranged from 43 to 80 years with a mean of 65.3 years. Tumor size was $3 \mathrm{~cm}$ or less in $16(32 \%)$ patients and greater than $3 \mathrm{~cm}$ in 34 (68\%) patients.

Histologically, 13 (26\%) tumors were of low grade and $37(74 \%)$ were of high grade. There were $8(16 \%)$ tumors of the Ta stage, $10(20 \%)$ of the T1 stage, 13 
Table 1 Clinicopathological features of the studied urothelial bladder carcinoma patients

\begin{tabular}{ll}
\hline & $\boldsymbol{N}(\%)$ \\
\hline Age & \\
$\leq 60$ years & $31(62 \%)$ \\
$>60$ years & $19(38 \%)$ \\
Sex & \\
Males & $38(76 \%)$ \\
Females & $12(24 \%)$ \\
Tumor size & \\
$\leq 3$ cm & $16(32 \%)$ \\
$>3$ cm & $34(68 \%)$ \\
Grade & \\
Low grade & $13(26 \%)$ \\
High grade & $37(74 \%)$ \\
PT stage & \\
Ta & $8(16 \%)$ \\
T1 & $10(20 \%)$ \\
T2 & $13(26 \%)$ \\
T3 & $1530 \%)$ \\
T4 & $4(8 \%)$ \\
AR expression & \\
Negative expression & $21(42 \%)$ \\
Positive expression & $29(58 \%)$ \\
\hline
\end{tabular}

Table 2 Correlation between AR expression and tumor grade in the studied urothelial bladder carcinoma patients

\begin{tabular}{llll}
\hline & $\begin{array}{l}\text { AR negative } \\
\text { patients } \\
\boldsymbol{N}(\%)\end{array}$ & $\begin{array}{l}\text { AR positive patients } \\
\boldsymbol{N}(\%)\end{array}$ & P value \\
\hline Low grade (13) & $8(61.5 \%)$ & $5(38.5 \%)$ & 0.07 \\
High grade (37) & $13(35.2 \%)$ & $24(64.8 \%)$ & \\
\hline
\end{tabular}

(26\%) of the T2 state, $15(30 \%)$ of the T3 stage and 4 (8\%) of the T4 stage.

Immunohisotchemical staining showed that AR was positively expressed in 29 (58\%) tumors form among all patients, while negative expression was observed in 21(42\%) patients.

The proportion of tumors positive for AR was higher in high-grade tumors than in low-grade tumors; however, this difference was statistically insignificant $(P=0.07)$ (Table 2). Also, no significant correlation was found between AR expression and the depth of tumor invasion (pT stage) $(P=0.09)$ (Table 3).Figures 1 and 2 show the expression of AR in low- and high-grade urothelial bladder carcinoma.
Table 3 Correlation between AR expression and pT stage in the studied urothelial bladder carcinoma patients

\begin{tabular}{llll}
\hline & $\begin{array}{l}\text { AR negative } \\
\text { patients } \\
\boldsymbol{N}(\%)\end{array}$ & $\begin{array}{l}\text { AR positive patients } \\
\boldsymbol{N}(\%)\end{array}$ & P value \\
\hline $\mathrm{Ta}(8)$ & $6(75 \%)$ & $2(25 \%)$ & 0.09 \\
$\mathrm{~T} 1(10)$ & $7(70 \%)$ & $3(30 \%)$ & \\
$\mathrm{T} 2(13)$ & $3(23.1 \%)$ & $10(76.9 \%)$ & \\
$\mathrm{T} 3(15)$ & $3(20 \%)$ & $12(80 \%)$ & \\
$\mathrm{T} 4(4)$ & $2(50 \%)$ & $2(50 \%)$ & \\
\hline
\end{tabular}

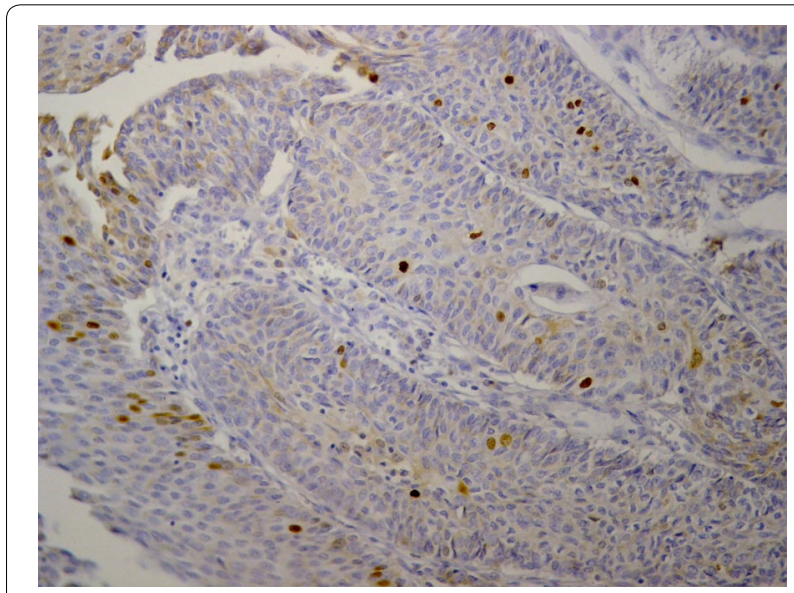

Fig. 1 Low-grade urothelial carcinoma showing few AR immunoreactive tumor cells $(<10 \%)(\times 200)$

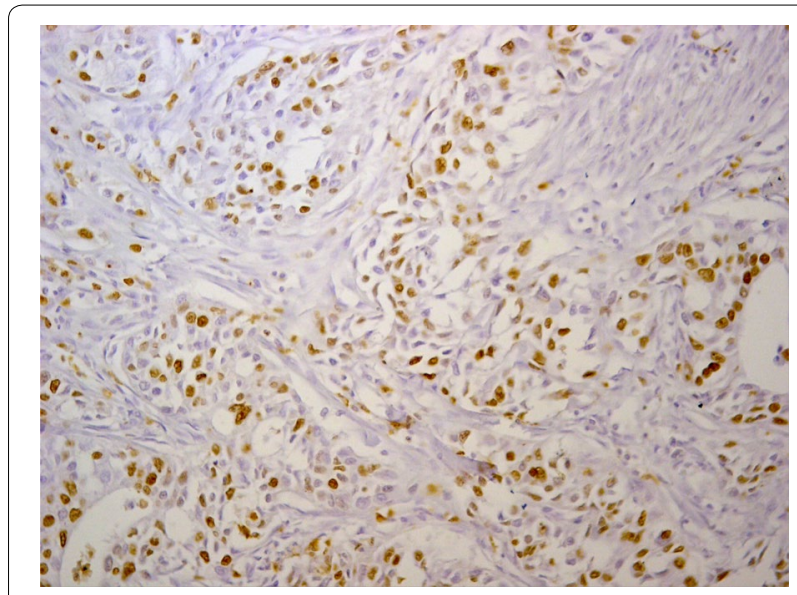

Fig. 2 High-grade urothelial carcinoma showing positive expression of $A R(>10 \%)(\times 200)$

\section{Discussion}

Bladder cancer is an international public health problem. According to GLOBOCAN 2012, developed countries 
and parts of Africa displayed higher incidence rates, while countries of North Africa and the Middle East had the highest mortality rates [13]. It is also observed that the incidence of bladder cancer is much higher in males than females [14]. This difference in both genders as well as the disparity in the natural history of the disease needs further research.

A considerable amount of clinical data indicates that steroid hormone receptor-mediated signals compose a critical part in urothelial tumorigenesis and tumor progression. Examples of these receptors are androgen receptors, estrogen receptors, progesterone receptors, glucocorticoid receptors and retinoid receptors. Actually, studies on urothelial cancer samples have verified that raised or decreased expression of these receptors or modifications of their pathways relates to patient outcomes. Thus, steroid hormone receptors and associated signals may serve as biomarkers for urothelial carcinoma and can feasibly predict tumor recurrence or progression [15]. Several of these studies have revealed the possible role of AR signaling pathway and its co-regulators on the progression and development of urothelial cancers anticipating the usage of anti-AR therapies for urothelial cancer patients $[16,17]$.

In Egypt until recently, urinary bladder cancer has been a commonly diagnosed cancer [18]. Searching for a link between the AR pathway and the Egyptian population, we investigated the AR expression in urothelial carcinoma of the urinary bladder of Egyptian cancer patients. We found that AR was expressed in 29 (58\%) of 50 evaluated tumors. This finding is close to that of Boorjian et al. [19] who reported AR expression in $53.1 \%$ of patients in their studied samples. However, different results were described by Miyamoto et al. [20], Nam et al. [9] and Mashhadi et al. [21] who detected the expression of AR in $42 \%, 37 \%$ and $22 \%$ of the bladder cancer patients, respectively. Such discrepancies between studies may be due to different staining conditions, criteria for over expression or patient populations.

In the current study, AR expression showed no statistically significant correlation with tumor grade $(P=0.07)$ and stage $(P=0.09)$. In this respect, it should be noted that previous studies that evaluated the relationship between AR and tumor aggressiveness of bladder cancer have led to conflicting results. For example, Tuygun et al. [12] reported a significant decrease in AR expression in higher grades and invasive tumors, which is consistent with the findings of Boorjian et al. [19]. In contrast, Mir et al. [22] showed that AR was elevated in muscle invasive tumors (15\%) compared to non-muscle invasive ones (9\%). In another study with 33 superficial bladder cancers, the authors concluded that patients with increased AR expression were more expected to have higher recurrence rates, compared to patients with lower AR expression [23]. Mashhadi et al. [21] detected a significant correlation between AR expression and high-grade and stage tumors. They also demonstrated higher rates of metastasis and lower relapse-free survival in AR positive patients compared to AR negative patients. In a different study, an inverse correlation was found between tumor grade and AR/androgen metabolizing enzymes expression [24].

Miyamoto et al. [20] stated that AR expression was significantly downregulated in high-grade muscle invasive tumors versus low-grade non-muscle invasive ones and found a significantly elevated expression of AR in lymph node metastasis as compared to primary tumors. In addition, it was found out that patients with AR positive muscle invasive tumors were apt to a higher risk of progression following radical cystectomy, although AR expression offered no prognostic discrimination regarding cancer-specific survival or tumor recurrence. Also, in another study, Ide et al. [15] reported that patients with AR positive non-muscle invasive tumors have a significantly lower risk of tumor recurrence compared to those with AR negative non-muscle invasive tumors, but not for disease progression.

A functional role for the AR in bladder cancer suggested that androgens inhibited bacilli Calmette-Guerininduced interleukin-6 expression in bladder cancer cell lines expressing the AR and that androgen deprivation therapy reversed this effect [25]. In an alternative study, Boorjian et al. [19] did not measure AR function directly, but they found that the localization of the AR to the nuclei of tumor cells implied that the ligand binding domain of the AR was intact and functional, as the AR was believed to translocate to the nucleus only after androgen binding.

Miyamoto et al. [23] investigated whether or not androgen regulates the progression of bladder cancer through AR. By means of cell proliferation assays and mouse xenograft models, they concluded that indeed androgen increased the growth of AR positive bladder cancer cells, while anti-androgen therapy inhibited cancer progression pointing out that proliferation of some bladder cancers was definitely androgen dependent. Additionally, they found out that AR knockdown using siRNA in AR-expressing bladder cancer cell lines also reduced cell proliferation, even in androgen-depleted environment, raising the possibility that AR signals (through androgenmediated and non-androgen-mediated mechanisms) might contribute to the promotion of bladder cancer progression. Furthermore, a prior in vivo study concluded that androgen-mediated AR signals can promote bladder carcinogenesis via down regulation of UDP-glucuronosyltransferases expression [26]. Also, it was found that 
conditional expression of AR increased the susceptibility to bladder cancer in mice [27].

\section{Conclusions}

In conclusion, our findings revealed the expression of AR in urothelial bladder carcinoma suggesting a possible role of androgen in bladder carcinogenesis. Targeting AR may provide novel chemopreventive and therapeutic approaches for bladder cancer.

\section{Authors' contributions}

MW contributed to the design, acquistion, analysis and drafting the manuscript. MK contributed to the conception, interpretation of data, drafting the manuscript and revising it. The authors approve the submitted form of the manuscript.

\section{Acknowledgements}

Not applicable.

\section{Competing interests}

The authors declare that they have no competing interests.

\section{Availability of data and materials}

The datasets used and/or analyzed during the current study are available from the corresponding author on reasonable request.

\section{Consent for publication}

Not applicable.

\section{Ethics approval and consent to participate}

The study was conducted in accordance with the ethics and guidelines provided by Faculty of Medicine, Beni-Suef University. The study involves a retrospective review of pathology blocks archived in the pathology Department, and according to institutional policies, such a study qualifies for a waiver of the local ethical review committee which was granted. The study does not involve direct or indirect contact with patients, and a consent to participate in the study was not required.

\section{Funding}

No specific source of funding was obtained. Work was conducted using facilities in Beni-Suef University.

\section{Author details}

1 Department of Pathology, Faculty of Medicine, Beni-Suef University, Beni-Suef 62521, Egypt. ${ }^{2}$ Unit of Pharmacology, Department of Tumor Biology, National Cancer Institute, Cairo University, Kasr Al Eini Street, Cairo 11796, Egypt.

Received: 11 October 2019 Accepted: 20 November 2019 Published online: 16 January 2020

\section{References}

1. Siegel R, Miller K, Jemal A (2016) Cancer statistics, 2016. CA Cancer J Clin 66:7-30

2. Ibrahim AS, Khaled HM, Mikhail NN, Baraka H, Kamel H (2014) Cancer incidence in Egypt: results of the national population-based cancer registry program. J Cancer Epidemiol 2014:437971

3. Burger M, Catto J, Dalbagni G, Grossman H, Herr H, Karakiewicz P, Kassouf W, Kiemeney L, Vecchia C, Shariat S, Lotan Y (2013) Epidemiology and risk factors of urothelial bladder cancer. Eur Urol 63:234-241

4. Ide H, Inoue S, Miyamoto H (2017) Histopathological and prognostic significance of the expression of sex hormone receptors in bladder cancer: a meta-analysis of immunohistochemical studies. PLoS ONE 12(3):e0174746

5. Sievert KD, Amend B, Nagele $U$ et al (2009) Economic aspects of bladder cancer: what are the benefits and costs? World J Urol 27:295-300

6. Siegel RL, Miller KD, Jemal A (2017) Cancer statistics 2017. CA Cancer J Clin 67(1):7-30
7. Siegel R, Naishadham D, Jemal A (2012) Cancer statistics. CA Cancer J Clin 62:10-29

8. Dobruch J, Daneshmand S, Fisch M, Lotan Y, Noon AP, Resnick MJ, Shariat SF, Zlotta AR, Boorjian SA (2016) Gender and bladder cancer: a collaborative review of etiology, biology, and outcomes. Eur Urol 69(2):300-310

9. Nam J, Park S, Lee S, Chung M (2014) Prognostic value of sex hormone receptor expression in non muscle invasive bladder cancer. Yonsei Med J 55:1214-1221

10. Eble JN, Sauter G, Epstein J et al (2004) World Health Organization classification of tumours. In: Pathology and genetics of tumours of the urinary system and male genital organs, chapter 2. Lyon: IARC Press

11. Greene FL, Page DL, Fleming ID et al (2002) AJCC Cancer staging manual, 6th edn. Springer, New York

12. Tuygun C, Kankaya D, Imamoglu A, Sertcelik A, Zengin K, Oktay M et al (2011) Sex specific hormone receptors in urothelial carcinomas of the human urinary bladder: a comparative analysis of clinicopathological features and survival outcomes according to receptor expression. Urol Oncol 29:43-51

13. Mahdavifar N, Ghoncheh M, Pakzad R, Momenimovahed Z, Salehiniya H (2016) Epidemiology, incidence and mortality of bladder cancer and their relationship with the development index in the World. Asian Pac J Cancer Prev 17(1):381-386

14. Yavari P, Sadrolhefazi B, Mohagheghi M, Mehrazin R (2009) A descriptive retrospective study of bladder cancer at a hospital in Iran (1973-2003). Asian Pac J Cancer Prev 10:681-684

15. Ide H, Miyamoto H (2015) Steroid hormone receptor signals as prognosticators for urothelial tumor. Dis Markers 2015:840640

16. Li P, Chen J, Miyamoto H (2017) Androgen receptor signaling in bladder cancer. Cancers (Basel) 9(2):20

17. Inoue S, Mizushima T, Miyamoto H (2018) Role of the androgen receptor in urothelial cancer. Mol Cell Endocrinol 465:73-81

18. Miyazaki J, Nishiyama H (2017) Epidemiology of urothelial carcinoma. Int J Urol 24(10):730-734

19. Boorjian S, Ugras S, Mongan N, Gudas L, You X, Tickoo S, Scherr D (2004) Androgen receptor expression is inversely correlated with pathologic tumour stage in bladder cancer. Urology 64:383-388

20. Miyamoto H, Yao J, Chaux A, Zheng Y, Hsu I, Izumi K, Chang C, Messing E, Netto G, Yeh S (2011) Expression of androgen and oestrogen receptors and its prognostic significance in urothelial neoplasm of the urinary bladder. BJU Int 109:1716-1726

21. Mashhadi R, Pourmand G, Kosari F, Mehrsai A, Salem S, PourmandM Alatab S, Khonsari M, Heydari F, Beladi L, Alizadeh F (2014) Role of steroid hormone receptors in formation and progression of bladder carcinoma: a case control study. Urol Oncol 11(6):1-6

22. Mir C, Shariat S, Van der Kwast T et al (2011) Loss of androgen receptor expression is not associated with pathological stage, grade, gender or outcome in bladder cancer: a large multi-institutional study. BJU Int 108:24-30

23. Miyamoto $\mathrm{H}$, Yang Z, Chen $\mathrm{Y}$ et al (2007) Promotion of bladder cancer development and progression by androgen receptor signals. J Natl Cancer Inst 99(7):558-568

24. Hata S, Ise K, Azmahani A, Konosu-Fukaya S, McNamara KM, Fujishima F, Shimada K, Mitsuzuka K, Arai Y, Sasano H, Nakamura Y (2017) Expression of AR, 5aR1 and 5aR2 in bladder urothelial carcinoma and relationship to clinicopathological factors. Life Sci 190:15-20

25. Chen F, Langenstroer P, Zhang G et al (2003) Androgen dependent regulation of bacillus Calmette-Guerin induced interleukin- 6 expression in human transitional carcinoma cell lines. J Urol 170:2009-2013

26. Izumi K, Zheng Y, Hsu J, Chang C, Miyamoto H (2013) Androgen receptor signals regulate UDP-glucuronosyltransferases in the urinary bladder: a potential mechanism of androgen induced bladder carcinogenesis. Mol Carcinog 52:94-102

27. Johnson DT, Hooker E, Luong R, Yu EJ, He Y, Gonzalgo ML, Sun Z (2016) Conditional expression of the androgen receptor increases susceptibility of bladder cancer in mice. PLoS ONE 11(2):e0148851

\section{Publisher's Note}

Springer Nature remains neutral with regard to jurisdictional claims in published maps and institutional affiliations. 